\title{
Levels of cell-free DNA and plasma KRAS during treatment of advanced NSCLC
}

\author{
ANNELI DOWLER NYGAARD ${ }^{1}$, KAREN-LISE GARM SPINDLER ${ }^{1}$, NIELS PALLISGAARD ${ }^{2}$, \\ RIKKE FREDSLUND ANDERSEN $^{2}$ and ANDERS JAKOBSEN ${ }^{1}$
}

\author{
Departments of ${ }^{1}$ Oncology and ${ }^{2}$ Clinical Biochemistry, Vejle Hospital, DK-7100 Vejle, Denmark
}

Received October 17, 2013; Accepted November 11, 2013

DOI: 10.3892/or.2013.2906

\begin{abstract}
Non-small cell lung cancer (NSCLC) is one of the most common malignant tumours in the Western world and is associated with a poor prognosis. Biomarkers predicting prognosis and therapeutic effects are highly required, and cell-free DNA (cfDNA) may be a feasible option. Genetic mutations can be analysed in plasma and may increase the scientific use of such measurements. In the present study, we investigated: i) the dynamics of cfDNA and plasma mutated KRAS (pmKRAS) during the treatment of patients with advanced NSCLC; and ii) the prognostic value of baseline cfDNA and pmKRAS. Sixty-nine patients were included in a prospective biomarker trial. Inclusion criteria included advanced NSCLC, candidate for first-line treatment, no previous cancer within the five years prior to this study. Blood samples were drawn at baseline, day 8 and at progression. Analyses of cfDNA and KRAS mutations in plasma were performed using an in-house qPCR assay. Evaluation of the treatment effect and status at follow-up was performed according to RECIST 1.1. The median levels of cfDNA were significantly higher at progression $(9,250$ alleles $/ \mathrm{ml})$ than at baseline $(5,450$ alleles $/ \mathrm{ml})$. Overall survival and progressionfree survival were both significantly shorter in patients with high levels of cfDNA (above the 75th percentile) compared to lower levels. Only few patients harboured KRAS mutations in plasma. Two patients had no KRAS mutations in plasma at baseline, but mutations appeared in the subsequent blood samples. High baseline levels of cfDNA indicate a poor prognosis. The level changes during the treatment course with a significant increase at progression, suggesting a possible predictive value of cfDNA. The plasma KRAS status may change during treatment with potential implications for treatment selection.
\end{abstract}

Correspondence to: Dr Anneli Dowler Nygaard, Department of Oncology, Vejle Hospital, Kabbeltoft 25, DK-7100 Vejle, Denmark E-mail: anneli.nygaard@rsyd.dk

Key words: cell-free DNA, circulating DNA, non-small cell lung cancer, blood-based biomarkers

\section{Introduction}

Lung cancer is one of the most common malignancies in the Western world and also in Denmark $(1,2)$. It is highly associated with smoking and, despite significant efforts in antismoking campaigns, the incidence is still increasing. Lung cancer can be sub-classified into small cell lung cancer (SCLC) and non-small cell lung cancer (NSCLC), with NSCLC being the most common (80\%). NSCLC can be further classified according to histology and genetic composition. The treatment of NSCLC comprises surgery, radiotherapy, chemotherapy and/or biological treatment, depending on the stage of disease, co-morbidity and the patient's general health. The disease may be cured by surgery, but the majority of patients are diagnosed at an advanced stage, leaving palliative treatment as the only option. Thus, the prognosis is often poor with only few patients experiencing long-term survival, calling for treatment improvements.

New treatment strategies are rapidly emerging and most are biological compounds with specific targets such as the epidermal growth factor receptor tyrosine kinase inhibitors (EGFR TKIs), which have proven highly effective in patients with activating EGFR mutations. Another treatment strategy are the EML4-ALK inhibitors effective in EML4-ALK positive lung cancer (3-6). Common characteristics for these genetic changes are that they are more frequent in adenocarcinomas and represent an early event in the development of cancer. Furthermore, it has become evident that new mutations may appear during the course of the disease, and the initial biopsy may, therefore, be insufficient as the basis for choice of treatment (7). Some of the mutations arising confer resistance to the current treatment (such as the EGFR T790M mutation causing resistance to EGFR TKIs) and identification of such mutations at an early stage may help tailor the treatment $(7,8)$. With the increasing knowledge of the complex genetic composition of NSCLC, mutation testing at baseline has gained significance.

To date, mutation analyses are generally performed on DNA derived from tumour tissue and, for practical reasons, mostly from the initial diagnostic biopsy. The new insights into the mutation status possibly changing throughout the treatment course necessitate repeated mutation analyses, leaving the clinicians with the dilemma of either performing multiple invasive procedures or staying on the path pointed out by the initial biopsy. Recent years have seen new and refined 
methods of identifying mutations in DNA derived from a blood sample, offering a highly appealing way of reducing the need for sequential invasive procedures.

The plasma mutated DNA represents a fraction of the cell-free DNA (cfDNA), which is small fragments of DNA circulating in the blood stream. The biological mechanisms underlying cfDNA remain to be fully understood, but increased apoptosis, necrosis of tumour cells, active release and lysis of circulating tumour cells (CTCs) have been proposed (9). cfDNA is present in both healthy individuals and patients with non-cancerous diseases, but the levels increase in patients with malignant diseases, probably due to increased activity of the aforementioned processes. Several studies have demonstrated a prognostic value of the total level of cfDNA, with high levels being associated with shorter survival (10-15). Furthermore, the qualitative perspective in terms of tumourspecific mutations has been investigated, demonstrating that it is indeed possible to identify tumour-specific mutations in the blood to serve as prognostic or predictive markers in different treatment settings $(16,17)$. The possibility of monitoring the disease opens new options calling for further investigation. Quantitative changes during the treatment course may predict the effect of the treatment and, thus, may represent a new tool for treatment monitoring, while qualitative changes such as new mutations in plasma are another important aspect $(17,18)$.

The present study investigated the relationship between the level of cfDNA and the disease development at different timepoints during the course of disease. Furthermore, the plasma KRAS mutation status during treatment and the prognostic impact of cfDNA was analysed.

\section{Materials and methods}

Patients. Patients with newly diagnosed, histopathologically confirmed stage III-IV NSCLC were included in a prospective biomarker study at the Department of Oncology, Vejle Hospital. Patients were included based on the following criteria: candidate for first-line treatment, age $>18$ years, no previous cancer diagnosis within five years before their inclusion in this study and written informed consent. The treatment comprised carboplatin [(GFR+25) x AUC mg i.v. on day 1] and vinorelbine $\left(30 \mathrm{mg} / \mathrm{m}^{2}\right.$ i.v. on day 1 and $60 \mathrm{mg} / \mathrm{m}^{2}$ p.o. on day 8$)$, alone or with bevacizumab $\left(7.5 \mathrm{mg} / \mathrm{m}^{2}\right.$ i.v. on day 1$)$.

Blood samples for analysis of cfDNA were drawn at baseline (within two weeks before treatment start), on day 8 of the first treatment cycle and at time of progression. The samples were stored at $-80^{\circ} \mathrm{C}$ until further analyses.

All patients had a baseline CT of the chest and upper abdomen within a month prior to treatment start. The treatment effect was evaluated by CT scans of the chest and upper abdomen and repeated at follow-up. Objective response rate was determined according to RECIST v.1.1 (19). The primary end-points were overall survival (OS) and progression-free survival (PFS).

The study was approved by the Regional Scientific Ethics Committee for Southern Denmark in accordance with the Danish law.

Plasma analyses of cfDNA and KRAS. A peripheral blood sample of $20 \mathrm{ml}$ collected in EDTA-tubes was drawn at each time-point and plasma was subsequently isolated by centrifugation at 2,000 x g for $10 \mathrm{~min}$. The plasma was separated within $2 \mathrm{~h}$ of blood sampling and subsequently stored at $-80^{\circ} \mathrm{C}$ until further analysis. Total nucleic acid was extracted from $1.0 \mathrm{ml}$ plasma by the use of a QIAsymphony virus/bacteria midi-kit on a QIAsymphony robot (Qiagen) according to the manufacturer's instructions. Both cfDNA and KRAS mutations were analysed by qPCR and an in-house developed assay. To determine the level of cfDNA a one-genome equivalent gene, the $\beta_{2}$ microglobuline (B2M) was used. The B2M-gene is not involved in carcinogenesis but is present in all cells and is therefore suitable for determining the total level of cfDNA regardless of cellular origin. The sequence of the forward primer was 3'-TAA AACTTAATGTCTTCCTTTTTTTTCTC-5', the reverse primer was 3'-AAACATTTTCTCAAGGTCAAAAACTTA-5', and the probe Fam-CCTCCATGATGCTGCTTACATGTC TC-Tamra. The rather small PCR product (102 bp) increases the sensitivity of the analysis, since smaller cfDNA fragments are more likely to be detected.

In order to prevent overestimation of the cfDNA caused by lysis of leukocytes or accidental mixing of leukocytes into the plasma, the samples were analysed for leukocyte contamination. Samples with evidence of leukocytes were excluded from the quantitative analyses.

The KRAS mutations were also analysed by in-house qPCR assays as previously described in detail $(14,20)$. In brief, six mutations of codon 12 and one in codon 13 of the KRASgene were identified by an ARMS-qPCR method. The high specificity of the method allowed for identification of KRAS mutations despite high background DNA.

All the reactions were run as doublets and the qPCR results were demonstrated as an amplification plot and subsequently transformed into alleles $/ \mathrm{ml}$ as previously described (14).

Statistical analysis. The correlations between patient characteristics, cfDNA and pmKRAS were investigated by non-parametric Wilcoxon rank sum test, $\chi^{2}$ test or Fischer's exact test when appropriate. The same tests were used for correlations between objective response rate and cfDNA level. Differences in level of cfDNA between different time-points of blood sampling were investigated by Wilcoxon rank-sum test. Survival analyses were evaluated by Kaplan-Meier plots and any differences between the groups were estimated by the log-rank method.

PFS was calculated as time between first day of treatment and objective progression or mortality due to any cause. OS was calculated as time between first day of treatment and date of mortality due to any cause. Patients with no events were censored by the last date of observation (May 15, 2013).

All statistical analyses were carried out in NSCSS statistical software (version 07.1.15 2009; NCSS Statistical Software, Kaysville, UT, USA). Two-sided P-value $<0.05$ was considered to indicate a statistically significant result.

\section{Results}

Patient selection. The present study included 69 patients. Patients with leukocyte contamination were excluded leaving 58 patients for analysis of cfDNA. The patient selection process is demonstrated in the consort diagram (Fig. 1) and patient 


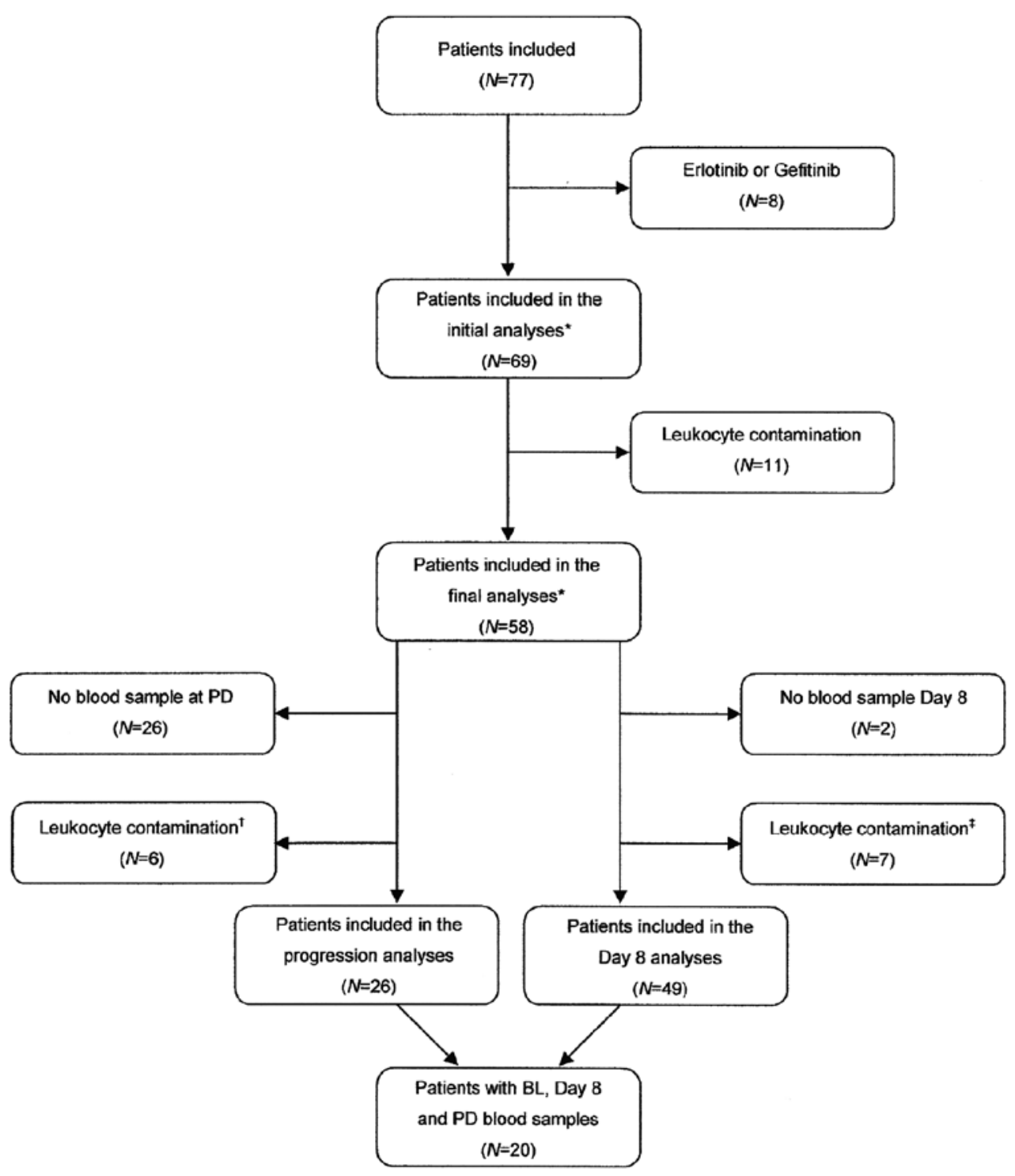

Figure 1. Consort diagram of the patient selection procedure. "Analyses of correlation between baseline cfDNA and prognosis (OS and PFS). 'Leukocyte contamination at progression or baseline. "Leukocyte contamination on day 8 or at baseline. Some patients did not progress before the end of the study period and were excluded and designated as the group of patients with 'no blood sample at PD'.

characteristics are provided in Table I. The patient characteristics were correlated with the baseline level of cfDNA. Patients with distant metastases (median cfDNA 7,100 alleles/ml) had a significantly higher level of cfDNA compared to patients without metastases (median cfDNA 4,900 alleles/ml; P=0.04). Furthermore, the patient characteristics were tested for differences in PFS and OS, but no significant differences were found in any of the variables.

Dynamics of cfDNA during treatment. When comparing the median level of cfDNA at baseline and time of progression $(\mathrm{n}=26)$, there was a significantly higher median at progression $(9,250$ alleles $/ \mathrm{ml})$ compared to baseline $(5,450$ alleles $/ \mathrm{ml})$; $\mathrm{P}=0.02)$. There were no significant differences between the level at baseline and on day 8 but a significantly higher level at progression compared to day 8 (data not shown).

Response was evaluated according to RECIST v.1.1, and the best overall response was correlated with the change in total cfDNA from baseline to day 8 . There was no obvious trend in the change of cfDNA depending on response. Due to the large differences in the level of cfDNA between the patients, the response was correlated with the size of change in terms of percent. There was no significant difference in response (PR, vs. NC vs. PD) between patients with an increase or decrease in cfDNA larger than either 25 or $50 \%$. There were no significant differences when the patients were grouped into stable disease $(\mathrm{PR}+\mathrm{NC})$ vs. $\mathrm{PD}$ either.

Fig. 2 illustrates the levels of cfDNA during the therapy in the 20 individual patients with cfDNA analyses available at all three time-points. Seventeen patients (85\%) had increasing values at progression. The difference between baseline (median cfDNA, 5,320 alleles $/ \mathrm{ml}$ ) and progression (median cfDNA, 9,250 alleles/ml) was significant $(\mathrm{P}=0.01)$.

Dynamics of pmKRAS during treatment. Only seven of the patients were identified with a plasma KRAS mutation at baseline (10\%). The mutation persisted during the treatment course. Two patients had changing mutation status during the 
Table I. Patient characteristics.

\begin{tabular}{|c|c|}
\hline Characteristics & Baseline values \\
\hline No. of patients, $\mathrm{n}(\%)$ & $58(100)$ \\
\hline \multicolumn{2}{|l|}{ Age (years) } \\
\hline Median (range) & $64(46-84)$ \\
\hline \multicolumn{2}{|l|}{ Gender, n (\%) } \\
\hline Male & $33(57)$ \\
\hline Female & $25(43)$ \\
\hline \multicolumn{2}{|l|}{ Histology, n (\%) } \\
\hline Adenocarcinoma & $48(83)$ \\
\hline $\mathrm{SCC}^{\mathrm{a}}$ & $8(14)$ \\
\hline Unclassified & $2(3)$ \\
\hline \multicolumn{2}{|l|}{ Stage, n (\%) } \\
\hline III & $6(10)$ \\
\hline IV & $52(90)$ \\
\hline \multicolumn{2}{|l|}{ Distant metastases ${ }^{\mathrm{b}}, \mathrm{n}(\%)$} \\
\hline Yes & $44(76)$ \\
\hline No & $14(24)$ \\
\hline \multicolumn{2}{|l|}{ CNS metastases, $\mathrm{n}(\%)$} \\
\hline Yes & $8(14)$ \\
\hline No & $50(86)$ \\
\hline \multicolumn{2}{|l|}{ ECOG PS ${ }^{c}$ at baseline, $\mathrm{n}(\%)$} \\
\hline 0 & $21(36)$ \\
\hline 1 & $29(50)$ \\
\hline 2 & $8(14)$ \\
\hline \multicolumn{2}{|l|}{$\mathrm{LDH}$ at baseline, $\mathrm{n}(\%)$} \\
\hline Normal & $33(57)$ \\
\hline Elevated & $22(38)$ \\
\hline Missing & $3(5)$ \\
\hline \multicolumn{2}{|l|}{ Treatment regimen, $\mathrm{n}(\%)$} \\
\hline Carboplatin + vinorelbine $(\mathrm{CN})^{\mathrm{d}}$ & $36(62)$ \\
\hline $\mathrm{CN}+$ bevacizumab & $22(38)$ \\
\hline
\end{tabular}

${ }^{\text {a }} \mathrm{SCC}$; squamous cell carcinoma; ${ }^{\mathrm{b}} \mathrm{Central}$ nervous system (CNS) metastases not included; ${ }^{\mathrm{C}}$ COG PS; Eastern Cooperative Oncology Group Performance Status; ${ }^{\mathrm{d}}$ one of the patients received vinorelbine as monotherapy.

treatment period. The first patient had no mutation at either baseline or day 8 , but at progression, and the second patient had no mutation at baseline but at day 8 and at progression.

Prognostic value of cfDNA. When investigating the prognostic value, the initial cut-off was set at quartiles, as previously described (12). By this exploratory approach, a significant difference between patients in the lower three quartiles and the upper fourth was observed ( $\mathrm{P}<0.05$; data not shown), leading to further dichotomisation by the upper fourth quartile.

This revealed a significantly shorter median OS of patients with high levels of cfDNA. The median survival time of all patients was 8.0 months (95\% CI, 6.7-9.3). Patients with high levels of cfDNA had a significantly shorter OS (median,
A
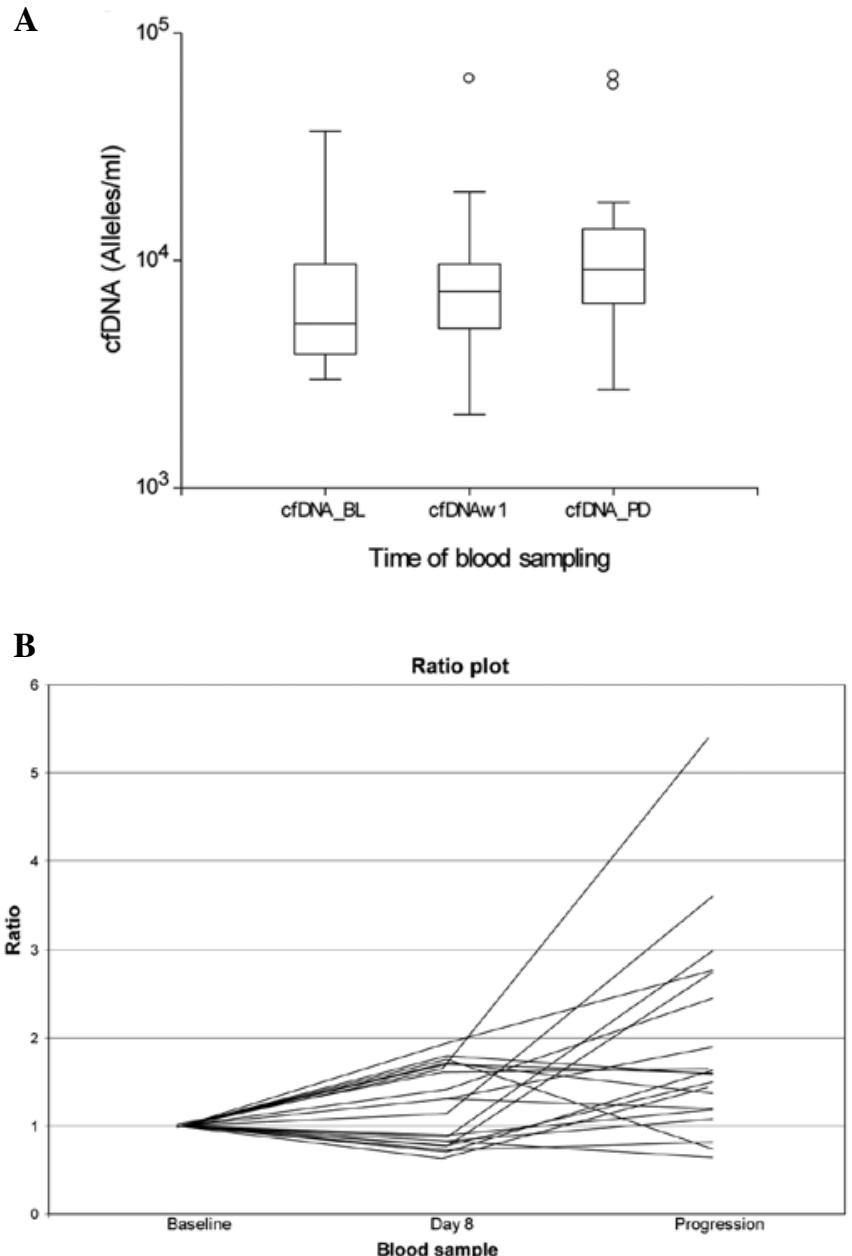

Figure 2. Box plot and ratio plot of the cfDNA level at different time-points. (A) The box plot illustrates the levels of cfDNA at baseline, day 8 and progression, and the P-values at the different time-points (Wilcoxon rank-sum test). (B) The ratio plot illustrates the changes in cfDNA from baseline to day 8 and at progression. Each line represents one patient $(n=20)$.

4.6 months; 95\% CI, 2.7-5.6) than patients with lower levels (median, 8.7 months; 95\% CI 7.9-11.4; HR, 3.1, 95\% CI 1.2-8.0; $\mathrm{P}=0.0004)$. Nineteen patients were censored. Survival curves are demonstrated in Fig. 3.

In addition to OS, PFS according to the baseline level of cfDNA was also investigated. As shown in Fig. 3, there were significant differences in PFS in patients with high vs. low levels of cfDNA. Eight patients were censored. The median PFS of patients with cfDNA $>75 \%$ was 2.6 months $(95 \% \mathrm{CI}$, 1.4-4.6) compared to 5.8 months $(95 \% \mathrm{CI}, 4.3-6.8)$ of the other patients (HR, 2.0; 95\% CI, 1.0-4.3; $\mathrm{P}=0.03$ ).

cfDNA at day $8(n=56)$ was not correlated with either PFS or OS (P>0.05; data not shown).

Prognostic value of plasma mutated KRAS (pmKRAS). pmKRAS was identified and quantified. Only 7/69 patients $(10 \%)$ had a mutation at baseline and further statistical analyses of the quantitative impact of pmKRAS were therefore not possible. Qualitative analyses were performed with the patients divided into two groups, with mutations or wild-type (WT). Nine patients were censored for PFS and 22 for OS. The median OS in the mutated group was significantly shorter than 

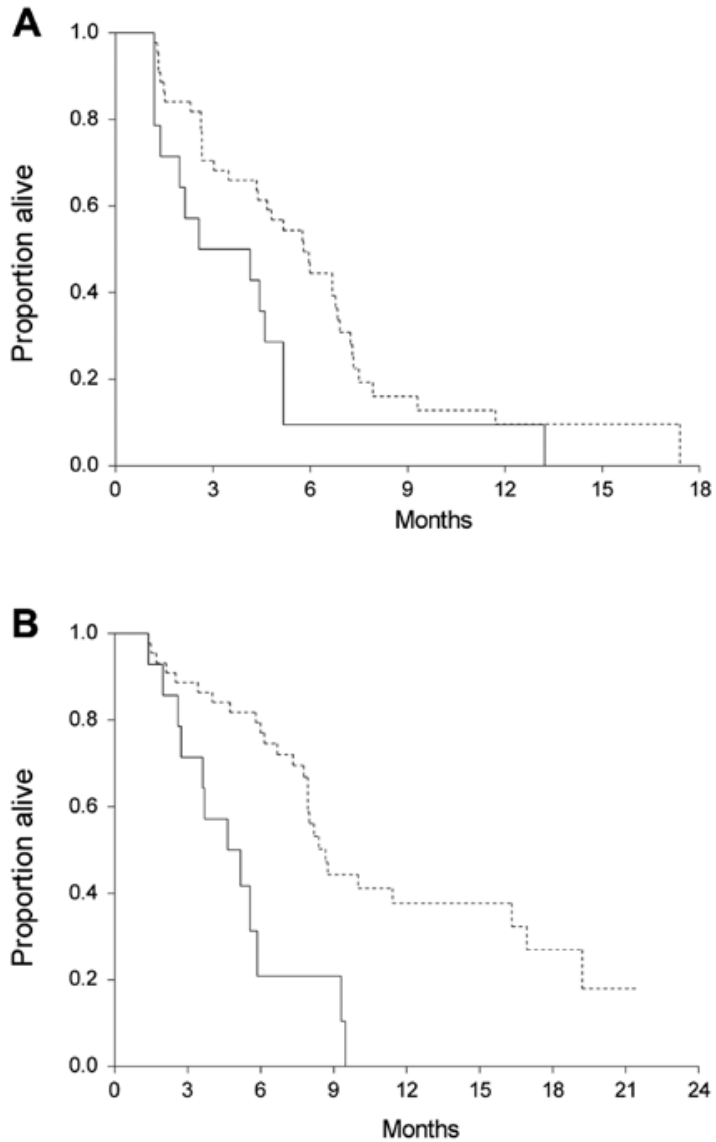

Figure 3. PFS and OS according to baseline level of cfDNA. (A) PFS $(\mathrm{n}=58)$. Median PFS $<75 \%, 5.8$ months (4.3-6.8); median PFS $>75 \%, 2.6$ months (1.4-4.6). HR, 2.0 (95\% CI 1.0-4.3); $\mathrm{P}=0.03$. (B) OS (n=58). Median OS $<75 \%, 8.7$ months (7.9-11.4); median OS $>75 \%, 4.6$ months (2.7-5.6). HR, 3.1 (95\% CI 1.2-8.0); $\mathrm{P}=0.0004$. The solid line represents patients with cfDNA in the $>75$ th percentile and the dotted line patients with cfDNA in the $<75$ th percentile.

in the WT group [median 3.6 (95\% CI, 2.1-4.3) vs. 8.4 months (95\% CI, 7.3-10.0; HR; 2.5 (95\% CI, 0.7-8.6); $\mathrm{P}=0.03$ ]. The same was observed in PFS, where patients harbouring a mutation had a median PFS of 2.1 months (95\% CI, 1.3-2.1) compared to 5.5 months $(95 \% \mathrm{CI}, 4.6-6.0)$ in the WT patients [HR, 2.6 (95\% CI, 0.8-8.6); $\mathrm{P}=0.01]$. Survival curves are shown in Fig. 4.

\section{Discussion}

The focus on and the need for sequential genetic testing throughout the course of the disease is rapidly increasing. Patient-friendly methods rather than invasive procedures are highly warranted, and the possibility of identifying reliable biomarkers and even tumour-specific mutations in a blood sample is highly advantageous. In the present study, we investigated both the level of cfDNA and the tumour-specific KRAS mutation in plasma at three different time-points. The material was prospectively collected by enrolment in a pre-defined biomarker protocol making the patient group homogenous and, importantly, also comparable with previous studies of the same area $(12,16)$.

The analyses were performed by an in-house developed assay, which has been used, validated and previously described
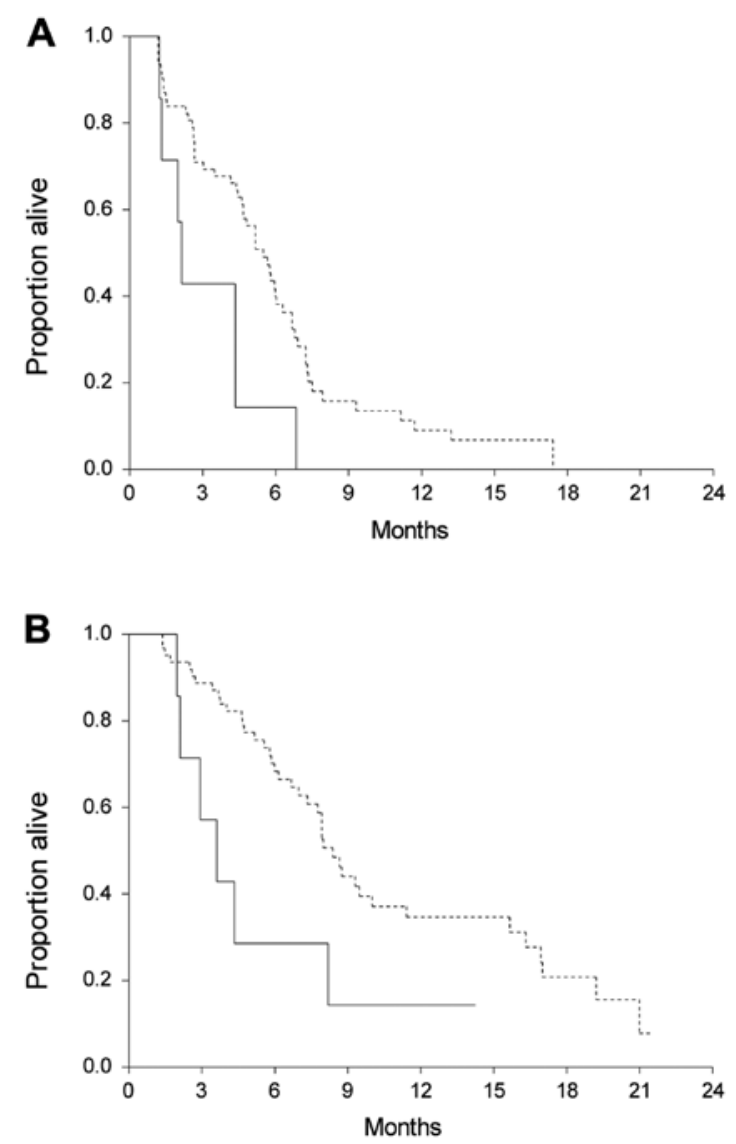

Figure 4. PFS and OS according to pmKRAS status [mutated or wild-type (WT)] at baseline $(n=69)$. (A) PFS in all patients $(n=69)$. Median PFS pmKRAS WT, 5.5 months (4.6-6.0); median PFS pmKRAS mutated, 2.1 months (1.3-2.1). HR, 2.6 (95\% CI, 0.8-8.6); $\mathrm{P}=0.01$. (B) OS in all patients $(\mathrm{n}=69)$. Median OS pmKRAS WT, 8.4 months (7.3-10.0); median OS pmKRAS mutated, 3.6 months (2.1-4.3). HR, 2.5 (95\% CI, 0.7-8.6); $\mathrm{P}=0.03$. The solid line represents patients with a KRAS mutation $(n=7)$, the dotted line WT patients $(n=62)$.

in detail $(14,16,20)$. The PCR-fragment used in the present study was short (only $102 \mathrm{bp}$ ), increasing the detection rate of even small fragments of cfDNA in the plasma. A method for identifying samples with possible leukocyte contamination was also used, enabling exclusion of patients with an unreliable estimation of the level of cfDNA.

The level of cfDNA changed significantly from baseline to progression, but not between baseline and day 8 , indicating a correlation between disease progression and increasing levels of cfDNA. Day 8 may be too early to detect any significant indications of the prognosis, but the same measurement at later time-points may be of interest in order to predict progression at an early stage. The issue has been investigated by other authors, although only few studies are available. In 2001, Sozzi et al (21) investigated the level of cfDNA before and after surgery and at follow-up of 38 patients with NSCLC. A reduction of cfDNA was seen in the relapse-free patients, while four patients had increasing levels in following measurements of cfDNA. Notably, all patients with increasing levels subsequently presented with cancer.

In agreement with our data, Kumar et al (22) also reported increasing levels of cfDNA in patients with progression after three courses of chemotherapy in 42 patients with advanced 
NSCLC. However, in contrast to our observations, the authors were not able to demonstrate any prognostic value of the baseline level of cfDNA as in the present study. Reasons for these discrepancies may be different cut-off limits as well as a heterogeneous patient population.

We examined the dynamics of pmKRAS at the different time-points. Ten percent of the patients had a KRAS mutation at baseline, which is lower than expected, but may be due to the relatively low number of patients included or, undetectable, low levels of KRAS in the circulation. Of note, two patients without detectable KRAS mutation at baseline were subsequently identified with KRAS mutations in the plasma. One of the patients had a mutation both on day 8 and at progression, while the other had a mutation only at time of progression. The findings may reflect limitations of the method in detecting KRAS mutations in plasma, tumour heterogeneity with increasing levels of the KRAS-mutated clone during treatment, or the appearance of new mutations. The same phenomenon has previously been demonstrated in metastatic colorectal cancer and is also discussed in a review by Mok (7), suggesting that new mutations may arise as a consequence of selection pressure during treatment (14).

In addition to the dynamics of cfDNA and pmKRAS, we investigated the prognostic value of the two parameters at baseline. We previously demonstrated an independent prognostic value of the level of cfDNA and the mutational status of pmKRAS in similar patient material and sought to validate these findings $(12,16)$. The same cut-off limits based on quartiles and subsequent dichotomisation at the 75 th percentile were used in the present study. We were able to validate the prognostic value of cfDNA both at the level of quartiles and at the 75th percentile, with higher levels of cfDNA being associated with a shorter OS and PFS. Furthermore, in our previous study, a subgroup analysis of patients with both high levels of cfDNA ( $>75$ th percentile) and a poor PS (PS=2) showed an even poorer prognosis for these patients. In the present study, only two patients fulfilled these criteria, leaving no room for further statistical analyses.

Our previous study showed an independent prognostic value of the presence of KRAS mutations in plasma, the mutations being associated with a poorer prognosis (16). Despite the low number of patients with pmKRAS in the present study, the results of our previous study were confirmed, indicating a prognostic value of pmKRAS in this setting.

cfDNA is a potentially valuable tool in monitoring treatment effect in patients with NSCLC, but the optimal time for analysis remains to be defined. Furthermore, tumour-specific mutations such as KRAS appear to be a dynamic phenomenon highlighting the need for repeated analyses of the tumour's genetic composition. The present results confirmed our previous findings, but further studies are required to outline the field of optimal clinical application of cfDNA and tumourspecific mutations measured in plasma.

\section{Acknowledgements}

The authors thank Yvette Schandorf Sørensen for keeping track of the blood sample collection and the dedicated co-workers of the Department of Biochemistry for technical assistance. We thank Karin Larsen for proofreading the final manuscript.

\section{References}

1. Engholm G, Ferlay J, Christensen N, et al: NORDCAN: Cancer Incidence, Mortality, Prevalence and Survival in the Nordic Countries, Version 5.3 (25.04.2013). Association of the Nordic Cancer Registries. Danish Cancer Society. http://www.ancr.nu. Accessed March 2013.

2. Ferlay J, Shin HR, Bray F, Forman D, Mathers C and Parkin DM: GLOBOCAN 2008 v2.0, Cancer Incidence and Mortality Worldwide: IARC CancerBase No. 10 [Internet]. International Agency for Research on Cancer, Lyon, 2010. http://globocan.iarc.fr. Accessed March 2013.

3. Hirsch FR, Janne PA, Eberhardt WE, et al: Epidermal growth factor receptor inhibition in lung cancer: status 2012. J Thorac Oncol 8: 373-384, 2013.

4. D'arcangelo M, Wynes MW and Hirsch FR: The role of anaplastic lymphoma kinase inhibitors in the treatment of advanced nonsmall cell lung cancer. Curr Opin Oncol 25: 121-129, 2013.

5. Sasaki T, Rodig SJ, Chirieac LR and Janne PA: The biology and treatment of EML4-ALK non-small cell lung cancer. Eur J Cancer 46: 1773-1780, 2010.

6. Soda M, Choi YL, Enomoto M, et al: Identification of the transforming EML4-ALK fusion gene in non-small-cell lung cancer. Nature 448: 561-566, 2007.

7. Mok TS: Personalized medicine in lung cancer: what we need to know. Nat Rev Clin Oncol 8: 661-668, 2011.

8. Kuang Y, Rogers A, Yeap BY, et al: Noninvasive detection of EGFR T790M in gefitinib or erlotinib resistant non-small cell lung cancer. Clin Cancer Res 15: 2630-2636, 2009.

9. Stroun M, Maurice P, Vasioukhin V, et al: The origin and mechanism of circulating DNA. Ann NY Acad Sci 906: 161-168, 2000.

10. Catarino R, Coelho A, Araujo A, et al: Circulating DNA: diagnostic tool and predictive marker for overall survival of NSCLC patients. PLoS One 7: e38559, 2012.

11. Gautschi O, Bigosch $\mathrm{C}$, Huegli $\mathrm{B}$, et al: Circulating deoxyribonucleic acid as prognostic marker in non-small-cell lung cancer patients undergoing chemotherapy. J Clin Oncol 22: 4157-4164, 2004.

12. Nygaard AD, Garm Spindler KL, Pallisgaard N, Andersen RF and Jakobsen A: Quantification of cell free DNA as a prognostic factor in advanced NSCLC. J Can Ther 4: 1-7, 2013.

13. Sirera R, Bremnes RM, Cabrera A, et al: Circulating DNA is a useful prognostic factor in patients with advanced non-small cell lung cancer. J Thorac Oncol 6: 286-290, 2011.

14. Spindler KL, Pallisgaard N, Vogelius I and Jakobsen A: Quantitative cell-free DNA, KRAS, and BRAF mutations in plasma from patients with metastatic colorectal cancer during treatment with cetuximab and irinotecan. Clin Cancer Res 18: 1177-1185, 2012.

15. van der Drift MA, Hol BE, Klaassen CH, et al: Circulating DNA is a non-invasive prognostic factor for survival in non-small cell lung cancer. Lung Cancer 68: 283-287, 2010.

16. Nygaard AD, Garm Spindler KL, Pallisgaard N, Andersen RF and Jakobsen A: The prognostic value of KRAS mutated plasma DNA in advanced non-small cell lung cancer. Lung Cancer 79: 312-317, 2013.

17. Punnoose EA, Atwal S, Liu W, et al: Evaluation of circulating tumor cells and circulating tumor DNA in non-small cell lung cancer: association with clinical endpoints in a phase II clinical trial of pertuzumab and erlotinib. Clin Cancer Res 18: 2391-2401, 2012.

18. Kumar S, Guleria R, Singh V, Bharti AC, Mohan A and Das BC: Plasma DNA level in predicting therapeutic efficacy in advanced nonsmall cell lung cancer. Eur Respir J 36: 885-892, 2010.

19. Therasse P, Arbuck SG, Eisenhauer EA, et al: New guidelines to evaluate the response to treatment in solid tumors. European Organization for Research and Treatment of Cancer, National Cancer Institute of the United States, National Cancer Institute of Canada. J Natl Cancer Inst 92: 205-216, 2000.

20. Steffensen KD, Waldstrom M, Grove A, Lund B, Pallisgard N and Jakobsen A: Improved classification of epithelial ovarian cancer: results of 3 danish cohorts. Int J Gynecol Cancer 21: 1592-1600, 2011.

21. Sozzi G, Conte D, Mariani L, et al: Analysis of circulating tumor DNA in plasma at diagnosis and during follow-up of lung cancer patients. Cancer Res 61: 4675-4678, 2001.

22. Kumar S, Guleria R, Singh V, Bharti AC, Mohan A and Das BC: Efficacy of circulating plasma DNA as a diagnostic tool for advanced non-small cell lung cancer and its predictive utility for survival and response to chemotherapy. Lung Cancer 70: 211-217, 2010 . 